\title{
Medium term growth forecasts: Experts vs. simple models
}

\author{
J. Daniel Aromí \\ IIEP Universidad de Buenos Aires-Conicet, FCE Universidad Católica, Argentina
}

\section{A R T I C L E I N F O}

\section{Keywords:}

GDP forecasts

Emerging countries

Simple models

Overreaction

Forecast evaluation

\begin{abstract}
A B S T R A C T
We compare the medium-term GDP growth forecasts generated by experts to those generated by simple models. This study analyzes a large set of forecasts that covers 48 countries from 1997 to 2016. Out-of-sample exercises indicate that no noticeable difference in performance is observed for advanced economies. In contrast, in the case of emerging economies, model forecasts perform better than expert forecasts. In addition, similar patterns are found for a collection of forecasts from a different set of experts, which suggests that the reported regularity is prevalent. Further analyses suggest that the documented difference in performance can be explained by an optimism bias, excessive reactions to innovations in growth trajectories, and insufficient responses to the information contained in the current account balance.
\end{abstract}

(c) 2019 International Institute of Forecasters. Published by Elsevier B.V. All rights reserved.

\section{Introduction}

How well do experts anticipate economic growth over multiple years? Can simple forecasting models outperform expert forecasts? Does the relative performance depend on the level of development of the economy under consideration? This work aims to address these questions in order to shed light on the properties of the mediumterm growth forecasts generated by experts.

Most important economic decisions (e.g. household saving, corporate investment, public debt issuance) are guided, to an important extent, by forecasts that span multiple years. Also, various prominent macroeconomic events such as debt crises are associated with errors in medium-term forecasts (Easterly, 2012; Heymann \& Sanguinetti, 1998). However, despite their relevance, medium-term forecasts have not received as much attention as short-term forecasts. More specifically, most of the literature has analyzed the properties of forecasts with prediction horizons of up to 24 months (Ager, Kappler, \& Osterloh, 2009; Dovern, Fritsche, Loungani, \& Tamirisa, 2015; Dovern \& Weisser, 2011; Loungani, 2001; Pons, 2000). While the evidence found in this literature

E-mail address: aromi.daniel@gmail.com. is informative about the probable properties of mediumterm forecasts, it is not known to what extent they share similar properties.

The present study evaluates expert forecasts by comparing their performances with those obtained by simple forecasting models that are estimated using historical information from a panel of countries. This type of evaluation is particularly convenient in the presence of structural shocks. In this case, experts need to learn the changing structure of the economy, and as a result, their forecasts tend to display correlated errors. Traditional evaluations of efficiency or conditional biases (e.g. tests à la Mincer \& Zarnowitz, 1969) could lead to the erroneous detection of inefficiencies (Batchelor, 2007; Dovern \& Weisser, 2011). In contrast, model forecasts face the same challenges as experts. That is, structural breaks need to be learned through a gradual adjustment of the forecasting model parameters.

The expert forecasts evaluated in this work were generated by the IMF's staff and published in multiple releases of the World Economic Outlook. The analysis focuses on five-year-ahead forecasts for 48 advanced and emerging countries covering the period from 1997 to 2016. Expert forecast are compared to predictions from autoregressive models that include at most two additional predictors. First, the GDP per capita is incorporated, to 
account for potential convergence patterns. Second, the current account balance is also considered, to account for external strengths or vulnerabilities that are not reflected in past growth performance or current wealth levels.

The results show that differences in forecast accuracy depend on the level of development of the economy. In the case of advanced economies, the accuracy of forecast models is very similar to that of expert forecasts. In sharp contrast, in the case of emerging economies, model forecasts are significantly more accurate. For emerging countries, the root mean square errors of model forecasts are between $9 \%$ and $22 \%$ below those associated with expert forecasts, depending on the model specification. Demonstrating the robustness of this finding, these differences in accuracy are also noticeable when the forecast errors are disaggregated by country or by year of forecast release.

A set of alternative forecast evaluations results in additional insights. It is found that forecasts based on time series regressions for individual countries are unable to produce similar performances. This finding suggests that the strong performances of simple forecasting models are based to an important extent on their ability to pool information regarding the past trajectories of multiple countries. In another forecasting exercise, iterative forecasts are shown to generate results that are similar to those reported in the original exercise. Similar differences in performance are observed when a collection of mediumterm forecasts from a private consulting firm is evaluated, suggesting that the reported finding might characterize the expectations of a broad set of experts. The case of short-horizon forecasts has also been considered. Simple models do not over-perform experts when current year or one-year-ahead prediction tasks are considered. As has been conjectured, the properties of medium-term forecasts differ from those of short-term forecasts.

Explanations for the differences in accuracy are explored in a second set of analyses. The resulting evidence suggests that the differences in performance can be explained by unconditional biases and heterogeneous responses to economic conditions. First, excessive optimism is detected in expert forecasts, but not in model forecasts. This finding is observed not only in the case of emerging economies, but also in the case of advanced economies. Second, expert forecasts display conditional errors that are associated systematically with growth accelerations and changes in the current account balance. Moreover, model forecasts respond differently to historic information. Model forecasts react more positively to an increment in the current account balance. In contrast, growth accelerations are associated with stronger increments in expected growth in the case of expert forecasts. This evidence is consistent with experts not being sufficiently attentive to the information provided by current account balances and attributing excessive persistence to innovations in growth rates.

As was reported by De Resende (2014), WEO's forecasts are generated by a mixture of subjective judgment and formal quantitative exercises. De Resende (2014) also reported that the use of quantitative tools is more intense in the case of advanced economies. Beyond this qualitative information, and in contrast to model forecasts, the process by which expert forecasts are generated is opaque or tacit. Hence, the available information does not allow us to determine the extent to which the different results observed in advanced and emerging economies are explained by disparities in the intensity with which these alternative methods are used. In any case, it must be noted that the careful design of the out-of-sample forecasting exercise ensures that the information used in the present study for a given forecast was available to experts at the time when that forecast was released. That is, this study shows that experts could have benefited from a more intense formal use of the available data.

The reported findings are related to a relatively small set of contributions that have analyzed medium-term forecasts of GDP growth. De Resende (2014) finds evidence consistent with an over-optimism bias in WEO forecasts. Ho and Mauro (2016) and Pritchett and Summers (2014) report indications of overreactions to recent growth performances. Aromí (2018) uses press sentiment indicators to document evidence that is consistent with an overreaction to information flows. Extending these contributions, the present study evaluates mediumterm forecasts by comparing their performances with those of simple forecasting models. We find a strong contrast between the properties of forecasts associated with emerging countries and those of advanced countries. While indications of inefficiencies have also been found in the case of advanced economies, simple models are only shown to be more accurate than expert forecasts in the case of emerging economies. To the best of our knowledge, this evidence has not been reported previously.

The findings documented in this study have implications for medium-term forecasting practice. The strong performance of model forecasts points to the value of simple frameworks that learn from regularities observed in multiple countries. Due to their low-frequency nature, medium-term forecast exercises suffer from small training datasets. In that context, the combination of data from multiple economies seems to be advantageous. At the same, the solid performance of models that propose different coefficients for advanced and emerging markets suggests that there are gains associated with the capture of heterogeneities.

Focusing on the properties of expert forecasts, a specific set of recommendations emerges. As has been documented, it is found that expert forecast would benefit from reductions in the manifested level of optimism. Second, the analyses suggest that more attention should be paid to the vulnerabilities and strengths communicated by current account balances. Finally, it is found that experts' accuracy levels would increase under a more agnostic view regarding the persistence of innovations to GDP growth rates.

The rest of the paper is organized as follows. The next section presents the methodology and the data. The main results are presented in Section 3. Section 4 focuses on explanations for the reported differences in performance. Section 5 concludes. 


\section{Data and methodology}

\subsection{Simple prediction models}

This study compares expert forecasts to forecasts generated by simple linear prediction models. These models are estimated recursively using an expanding set of historic information for the panel of countries sampled.

The use of simple models is, in part, a consequence of the absence of rich economic information for a substantial set of emerging economies over long periods. Also, simple models can be viewed as conservative benchmarks that can be used to evaluate inefficiencies in expert forecasts. That is, simple model forecasts establish a lower bound on the level of inefficiency. Finally, simple models allow for a straightforward evaluation of the information provided by the predictors proposed in each specification.

We consider three models. The first and simplest model (Model 1) presents four predictors. Under this spartan specification, the expected cumulative GDP growth is a function of previous growth outcomes in three preceding non-overlapping five-year periods and a metric of wealth. Formally, the model is given by:

$$
\begin{aligned}
g_{c[t+1, t+5]}= & \alpha+\sum_{k \in\{5,10,15\}} \beta_{k} g_{c[t-k, t-k+4]} \\
& +\beta_{g d p p c} g d p p c_{c, t-1}+u_{c, t},
\end{aligned}
$$

where $g_{c[t+s, t+s+4]}$ denotes the cumulative GDP growth for country $c$ during the five-year period $[t+s, t+s+4]$, $\operatorname{gdppc}_{c, t-1}$ is the ratio of country c's GDP per capita in year $t-1$ to the US GDP per capita in the same year, and $u_{c, t}$ is an error term.

The model captures differences in information content by allowing for different coefficients for growth outcomes with different lags. Following standard practice, ${ }^{1}$ a wealth metric is incorporated to capture convergence patterns in historical data that might contain useful information regarding future growth.

The second model (Model 2) incorporates the current account balance as an additional predictor. More specifically, the new predictor is the current account balance in year $t-1$, expressed as fraction of GDP. The current account balance is included as a predictor because it might reflect vulnerabilities or strengths that may influence future GDP growth levels. An ample body of literature has studied current account sustainability, current account reversals, and their implications for economic growth. ${ }^{2}$ While current account deficits do not necessarily lead to negative assessments, ${ }^{3}$ an important fraction of the relevant literature has linked current account deficits to crisis episodes that have substantial negative implications for growth outcomes. For example, Edwards (2004) finds that current account reversals result in an average drop

\footnotetext{
1 See for example Barro (1991).

2 See for example Calvo (1998), Edwards (2004), Milesi-Ferreti and Razin (1996), Obstfeld and Rogoff (2007) and Reinhart and Reinhart (2008).

3 See for example the views expressed by Blanchard and Giavazzi (2002) and Sachs (1981).
}

of approximately $2.5 \%$ in the same year GDP growth. Another reason for including the current account balance as a predictor is that, unlike other variables that might be used to explain growth (e.g. human capital or institutional quality), the information provided by the current account balance is probably not reflected in the lagged economic growth. More specifically, vulnerabilities associated with potential current account reversals are not captured by past GDP growth performances. Model 2 is given by:

$$
\begin{aligned}
g_{c[t+1, t+5]}= & \alpha+\sum_{k \in\{5,10,15\}} \beta_{k} g_{c[t-k, t-k+4]} \\
& +\beta_{g d p p c} g d p p c_{c, t-1}+\beta_{c a b} c a b_{c, t-1}+u_{c, t-1},
\end{aligned}
$$

where $c a b_{c, t-1}$ is the current account balance for country $c$ in year $t-1$, expressed as a fraction of GDP.

Finally, we consider a more flexible model. Model 3 allows for different forecasting equations depending on whether the country is classified as advanced or emerging, with the aim of generating predictions that contemplate the probable heterogeneous associations between outcomes and predictors. In this spirit, Model 3 is given by two equations that match the specification used in Model 2: one equation is used to fit and predict GDP growth for advanced economies, while the other is used to fit and predict GDP growth for emerging economies. It is worth noting that, while proposing a flexible model might result in gains due to heterogeneous associations being captured, it might also result in an inferior performance due to the inefficiencies associated with a greater number of estimated parameters. In the end, this is a discussion that is resolved empirically.

\subsection{Data}

This study analyzes forecasts that cover 48 countries from 1997 to 2016. Each sampled country is classified as either advanced or emerging, with the classification being based on the categorization presented in the World Bank's 1990 World Development Report. The countries in the high-income group are classified as advanced, while those in the other groups are classified as emerging. This classification results in 21 advanced countries and 27 emerging countries. The list of countries in each group can be found in Appendix A.

The expert forecasts used in this work correspond to those available through the IMF's World Economic Outlook historical forecasts database. ${ }^{4}$ The database provides information on forecasts released in each April and October. In this study, only forecasts released in April are used. For each country and year combination, we compute fiveyear cumulative GDP growth forecasts for years $t+1$ to $t+5$.

A real-time database is built for training forecasting models and generating model forecasts. Three variables are used: GDP growth, GDP per capita, and current account balance as a fraction of GDP. For the three variables,

\footnotetext{
4 Available at: https://www.imf.org/external/pubs/ft/weo/data/WEO historical.xlsx.
} 
a careful construction of the database guarantees that all of the information used to train the models and generate model forecasts was available at the time of the release of the matching expert forecasts.

The GDP growth data from 1961 to 1987 are from Penn World Table 5.6 (PWT 5.6), released in January 1995 (Heston, Summers, Nuxoll, \& Aten, 1995); that is, 15 months before the release of the first collection of forecasts analyzed in the exercise below. The GDP growth data for the following years (starting with 1988) are from the World Economic Outlook's (WEO's) Historical Forecasts Database. In addition to GDP growth forecasts, this database also provides data on GDP growth realizations that were gathered by IMF staff at the time of the WEO exercises. More specifically, the database provides realtime GDP growth data corresponding to the two years that precede the year of the forecast release. These historical GDP growth data were available at the time of forecast release. This addresses any concerns regarding forward-looking biases.

The GDP per capita data until 1992 are from PWT 5.6. The information after 1992 is from various issues of the World Bank's World Development Report. ${ }^{5}$ We avoided the introduction of a forward-looking bias into the model forecasts by requiring the GDP per capita figures that were entered for a given year to be available before the release of the WEO's forecasts in April of the following year.

Current account balance data are also provided by multiple sources. Data on the current account balance from 1975 to 1987 were collected manually from various United Nations' Statistical Yearbooks. ${ }^{6}$ These figures were expressed as a fraction of GDP using PWT 5.6 data on current dollars GDP. The data on the current account balance from 1988 onward are from the WEO's Historical Forecasts Database. As in the case of the GDP growth figures, no forward-looking bias is introduced because the figures for the current account balance that corresponded to each year were gathered by IMF staff for the forecasting exercises released in April of the following year.

Table 1 shows descriptive statistics for the data used in this study. In addition to the original data, it also shows descriptive statistics for forecast errors, squared forecast errors and absolute errors. It should be noted that the sample standard deviation of forecast errors is 0.107 . This figure illustrates the importance of errors associated with medium-term forecasts.

\section{Model estimation and results}

Each forecasting model was estimated using an expanding set of information. For each year $t \in\{1996, \ldots$, 2011\}, models were estimated by exploiting the past association between the cumulative growth rates for five-year windows and the corresponding predictors. The model lag structure implies that GDP growth data as far as 15 years

\footnotetext{
5 Available at: http://www.worldbank.org/en/publication/wdr/wdrarchive.

6 The yearbooks can be downloaded from: https://unstats.un.org/ unsd/publications/statistical-yearbook/past-issues/.
}

Table 1

Descriptive statistics.

\begin{tabular}{lrllll}
\hline & $N$ & Mean & St. Dev. & Min & Max \\
\hline Expert forecasts & 1249 & 0.211 & 0.102 & -0.082 & 0.610 \\
GDP growth & 1946 & 0.181 & 0.148 & -0.431 & 0.886 \\
Forecast errors & 959 & -0.048 & 0.107 & -0.449 & 0.358 \\
Squared forecast errors & 959 & 0.014 & 0.024 & 0.000 & 0.202 \\
Absolute forecast errors & 959 & 0.088 & 0.077 & 0.000 & 0.449 \\
GDP per capita & 1711 & 0.436 & 0.343 & 0.008 & 2.423 \\
Current account balance & 1896 & -0.002 & 0.058 & -0.223 & 0.416 \\
\hline
\end{tabular}

Notes: The GDP growth and GDP growth forecasts are expressed as cumulative growth rates for five-year windows. The GDP per capita is expressed as a fraction of the US level. The current account balance is expressed as a fraction of GDP.

back is needed in order to construct a valid training observation. The training sample starts in 1976 and includes information on economic growth starting in 1961. In addition, some recent information cannot be incorporated into the training sample so as to avoid forward-looking biases. More specifically, to be included in the training dataset, the target five-year windows must start at least six years before the year of the corresponding model forecast exercise. As a result, the training data observations for each year in which model forecasts are generated, $t \in\{1996, \ldots, 2011\}$, include cumulative five-year growth realizations for windows $\left[t^{\prime}+1, t^{\prime}+5\right]$, where $t^{\prime}$ belongs to $\{1976, \ldots, t-6\}$.

Table 2 shows the estimated parameters for each forecasting model in the case of the last prediction task; that is, the fitted values based on the training sample used to generate forecasts in the year 2011. As expected, the estimations indicate a positive association between lagged growth and subsequent growth outcomes. In most cases, the coefficients decrease with the temporal distance. On the other hand, the estimated coefficient for lagged growth rates are low, and in many cases are not statistically significant. For example, in the case of Model 1 , the sum of the estimated coefficients is approximately 0.3 . This suggests a positive but weak link between past growth performances and expected growth performances. The equations fitted for Model 3 suggest that the lagged growth is more informative about subsequent growth in the case of emerging countries.

Consistent with the presence of convergence patterns, the estimations show a negative relationship between the GDP per capita and subsequent growth levels. According to the estimated coefficients for Model 3, in the case of advanced economies, a 10\% increment in GDP per capita (as a fraction of the US level) is associated with a fall of $1.5 \%$ in expected cumulative growth over the subsequent five-year period. In the case of emerging economies, the associated fall in expected growth is $4 \%$.

Models 2 and 3 indicate a positive association between the current account balance and the growth in subsequent five-year windows. The estimation of Model 3 indicates that the positive association is particularly noticeable in the case of emerging economies. In this case, a $1 \%$ increment in the current account balance (as a percentage of GDP) is associated with a $0.68 \%$ increment in the expected cumulative GDP growth over the subsequent five-year period. In other words, current account 
Table 2

Estimated forecasting models.

\begin{tabular}{lllll}
\hline & {$[1]$} & {$[2]$} & [3: Adv.] & [3: Em.] \\
\hline$\alpha$ & $0.168^{* * *}$ & $0.181^{* * * *}$ & $0.208^{* * *}$ & $0.214^{* * *}$ \\
& $(6.7)$ & $(7.3)$ & $(5.0)$ & $(6.9)$ \\
$\beta_{t-5, t-1}$ & $0.125^{* * *}$ & $0.125^{* * *}$ & 0.14 & $0.130^{* * *}$ \\
& $(2.4)$ & $(2.3)$ & $(1.2)$ & $(2.5)$ \\
$\beta_{t-10, t-6}$ & $0.122^{*}$ & $0.102^{*}$ & 0.032 & $0.133^{* *}$ \\
& $(1.9)$ & $(1.7)$ & $(0.4)$ & $(2.3)$ \\
$\beta_{t-15, t-11}$ & 0.049 & 0.050 & $0.068^{*}$ & 0.061 \\
$\beta_{g d p p c}$ & $(1.1)$ & $(1.1)$ & $(1.7)$ & $(0.9)$ \\
& $-0.120^{* * * *}$ & $-0.135^{* * *}$ & $-0.151^{* * *}$ & $-0.399^{* * *}$ \\
$\beta_{\text {cab }}$ & $(-4.4)$ & $(-5.1)$ & $(-3.7)$ & $(-4.1)^{* * *}$ \\
& - & $0.321^{*}$ & 0.144 & $0.681^{* *}$ \\
$N$ & & $(1.7)$ & $(1.0)$ & $(3.4)$ \\
$R^{2}$ & 1282 & 1282 & 612 & 670 \\
& 0.214 & 0.229 & 0.179 & 0.230
\end{tabular}

Notes: The estimated coefficients correspond to the models used to generate predictions in the year 2011. The $t$-statistics (in parentheses) were computed using year and country clusters, following Cameron, Gelbach, and Miller (2011). ", ${ }^{* *}$ and ${ }^{* * *}$ indicate significance at the $10 \%$, $5 \%$, and $1 \%$ levels, respectively.

surpluses and deficits seem to carry more news in the case of emerging economies. This is consistent with the empirical literature on current account sustainability. ${ }^{7}$ Additional exercises, reported in Appendix C, show that the estimated parameters are quite stable.

\subsection{Comparing forecast performances}

The forecast accuracies of expert forecasts and model forecasts are approximated by computing root mean square errors (RMSEs). The first row in Table 3 shows RMSEs for expert forecasts. As an initial observation, it can be noted that the accuracy is substantially higher in the case of advanced economies. While the RMSE computed in the case of advanced economies is 0.085 , that in the case of emerging economies is more than $50 \%$ higher, at 0.134 .

Regarding the comparison with model forecasts, again, there is a strong contrast between the cases of advanced and emerging economies. In the first case, the model forecast accuracy is similar to the expert forecast accuracy. The model forecasts' RMSEs are slightly lower than those of the expert forecasts in the cases of Models 1 and 2. In the case of Model 3, the RMSEs are almost identical. Independently of the model specification, tests for differences in accuracy indicate that the differences are not significant. However, given the simplicity of the forecasting models and the plausibly superior information available to experts, this similar level of accuracy observed in the case of advanced countries points to the existence of inefficiencies in the experts' forecasts. Tentatively, this evidence suggests that, in the case of advanced countries, expert forecasts could benefit from the information captured by model forecasts. ${ }^{8}$

\footnotetext{
7 See for example Reinhart and Reinhart (2008).

8 In this direction, an untabulated optimal forecast combination exercise indicates that the optimal weights are 0.8 for expert forecasts and 0.2 for Model 3 forecasts.
}

Table 3

Comparison of accuracy levels.

\begin{tabular}{llll}
\hline & All & Adv. countries & Emerg. countries \\
\hline $\begin{array}{l}\text { A. Expert forecasts } \\
\text { RMSE }\end{array}$ & 0.114 & 0.085 & 0.134 \\
B. Model 1 & & & \\
RMSE & 0.106 & 0.082 & 0.122 \\
Ratio (B/A) & 0.923 & 0.967 & 0.908 \\
$t$-statistic & -1.48 & 0.23 & -1.68 \\
$p$-value & 0.138 & 0.815 & 0.093 \\
C. Model 2 & & & \\
RMSE & 0.103 & 0.080 & 0.119 \\
Ratio (C/A) & 0.902 & 0.945 & 0.887 \\
$t$-statistic & -1.62 & 0.25 & -1.85 \\
$p$-value & 0.105 & 0.806 & 0.064 \\
D. Model 3 & & & \\
RMSE & 0.096 & 0.085 & 0.105 \\
Ratio (D/A) & 0.841 & 1.003 & 0.780 \\
$t$-statistic & -2.14 & 1.06 & -2.76 \\
$p$-value & 0.032 & 0.291 & 0.006 \\
\hline
\end{tabular}

Notes: The table shows $t$-statistics and $p$-values for two-tailed tests of forecast accuracy differences, as proposed by Pesaran, Schuermann, and Smith (2009). The tests are computed using a subsample of forecasts for non-overlapping five-year windows.

In contrast to the case of advanced countries, model forecasts result in noticeable improvements in accuracy when emerging countries are considered. Depending on the model specification, the model forecast RMSEs are between $9 \%$ and $22 \%$ below the expert forecast RMSEs, and the accuracy levels are judged to be statistically different in all cases, with different levels of significance. The bestperforming forecasts are those from Model 3. In this case, the computed RMSE is 0.105 . That is, the model forecast RMSE is $22 \%$ lower than the expert forecast RMSE. The relative performances across the three models indicate that the current account balance provides valuable information. Also, it points to gains associated with forecasting models that treat emerging and advanced countries differently.

A more disaggregated description of relative forecast accuracies can allow for additional insights, and at the same time serve as a robustness test of the results reported above. For example, it can reveal the existence of outliers that drive certain results. First, RMSEs are computed for individual economies. Fig. 1 summarizes this information in the form of histograms. The reported metric is the difference between the expert forecast RMSE and those of the model forecasts. A positive number indicates that the model forecasts show a higher accuracy. In the case of advanced countries, the figures show a marked similarity between the performances of expert forecasts and model forecasts. Differences in accuracy across countries are distributed more or less evenly around zero. For example, in the case of Model 3 forecasts, expert forecast are more accurate in 11 out of 21 countries. In addition, the difference in performance for most countries (20 out of 21 ) is between -0.02 and 0.02 .

In the case of emerging countries, histograms show more dispersion and a shift towards larger values. Independently of the model specification, the mode is to the right of zero, and expert forecasts are found to be more 
accurate in, at most, six out of 27 countries. In the case of Model 3, the expert forecasts underperform the model forecasts by more than 0.02 in 20 out of 27 economies. These observations serve as strong evidence that model forecasts are more accurate than expert forecasts for this second group of economies. Also, it is worth noting that there is no outlier tilting the result in favor of model forecasts.

A complementary description involves comparing the average forecast accuracy by year of forecast release. This might shed light on the extent to which the differences in performance are concentrated on specific years. Fig. 2 shows RMSEs by the year of forecast release, distinguishing between advanced and emerging economies. In the case of advanced countries, the expert forecast and the model forecasts follow very similar trajectories, independent of the model specification. For emerging countries, the relative performance of the model forecast improves between Model 1 and Model 3. Important differences in performance are observed in the early sample years for all models, as well as for forecasts generated in the late sample years when Model 3 is considered. Overall, these descriptive analyses serve as further support for the findings on relative forecast performances reported in Table 3.

\subsection{Private sector forecasts}

So far, we have reported findings regarding mediumterm GDP growth forecasts generated by IMF staff. We have established that simple models outperform experts in the case of emerging markets, and there are reasons to suspect that this property is not exclusive to WEO's forecasts. Social learning and the likely use of a common set of prediction tools can lead to similar forecasts for a broad set of economic actors. In addition, the previous literature has shown that different experts tend to generate similar forecasts (Loungani, 2001; Timmermann, 2007)

We therefore built a database of forecasts from a private consulting firm, with the aim of evaluating the medium-term forecasts released by another set of experts. More specifically, the medium-term forecasts correspond to those released by the Economist Intelligence Unit (EIU), the research and analysis division of The Economist Group.

The database consists of EIU's growth forecasts for non-overlapping five-year windows. Each prediction was collected manually from publications distributed by the consulting firm, ${ }^{9}$ and a total of 128 forecasts were extracted, corresponding to non-overlapping five-year windows starting in the years 1998, 2003 and 2008. The target of each forecast is the cumulative GDP growth during the current year and the subsequent four years. While the sample size is small compared to the WEO historical forecasts database studied earlier, we use this information to obtain a first assessment of the relative forecast performances of an alternative set of experts.

Table 4 provides information on the performance of the EIU's forecasts. It also allows a precise comparison of the experts' accuracy by presenting the performance

\footnotetext{
9 The publications were accessed using the ProQuest database.
}

Table 4

Comparison of accuracy levels: Economist Intelligence Unit and WEO.

\begin{tabular}{llll}
\hline & All & Adv. countries & Emerg. countries \\
\hline EIU forecasts: & & & \\
A. Expert RMSE & 0.111 & 0.089 & 0.127 \\
B. Model RMSE & 0.094 & 0.074 & 0.109 \\
Ratio (B/A) & 0.850 & 0.830 & 0.859 \\
$t$-statistic & -1.23 & -0.91 & -1.32 \\
$p$-value & 0.217 & 0.363 & 0.188 \\
$N$ & 128 & 60 & 68 \\
WEO forecasts: & & & \\
A. Expert RMSE & 0.105 & 0.066 & 0.130 \\
B. Model RMSE & 0.094 & 0.074 & 0.109 \\
Ratio (B/A) & 0.898 & 1.112 & 0.841 \\
$t$-statistic & -1.58 & 0.70 & -1.79 \\
$p$-value & 0.113 & 0.481 & 0.073 \\
$N$ & 128 & 60 & 68 \\
\hline
\end{tabular}

Notes: The table shows $t$-statistics and $p$-values for two-tailed tests for forecast accuracy differences, as proposed by Pesaran et al. (2009). The results are based on a sample of non-overlapping five-year forecasts from EIU, together with a matching sample from WEO. The model forecasts are those from Model 3.

of a matching sample of 128 of WEO's forecasts as well. Finally, information on the performance of Model 3 is reported too. Consistent with the previous analysis of WEO's forecasts, the RMSEs of the model forecasts are lower than those corresponding to EIU's forecasts. In the case of emerging economies, the model forecast RMSEs are $14 \%$ smaller. This difference is similar to that observed for the matching WEO forecasts. Nevertheless, the test for differences in accuracy levels fails to reject the null hypothesis of no difference, probably due to the small number of observations and the high variability in EIU's squared forecast errors. In the case of advanced economies, the performance of EIU's forecasts is worse than those of either the WEO's forecasts or the model forecasts. While the difference is not statistically different from zero, the accuracy metrics hint at a larger set of countries for which models are seen to perform better than experts. In summary, this simple exercise serves as an indication that the previously reported evidence on expert forecasts is a property that can be expected to characterize the forecasts issued by a diverse set of experts.

\section{Explaining differences in performance}

Having documented differences in forecast accuracy, this section gains additional insights through a series of descriptive analyses. These analyses are intended to identify characteristics of expert and model forecasts that can account for the differences in accuracy. The analysis starts by focusing on unconditional errors and looking for evidence that is consistent with an optimism bias. Next, we document conditional biases in expert forecasts. These biases are rationalized in the light of differential responses to relevant economic information. Finally, we report differences in relative accuracy conditional on cumulative growth realizations. 
Model 1
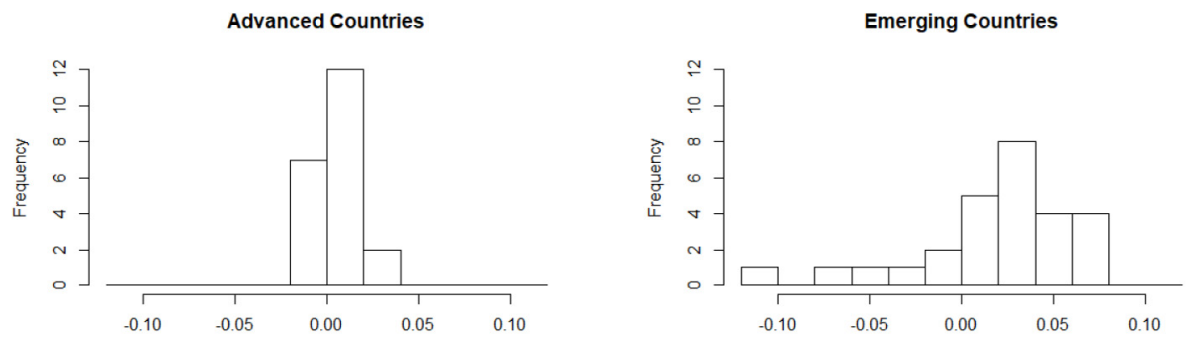

Model 2
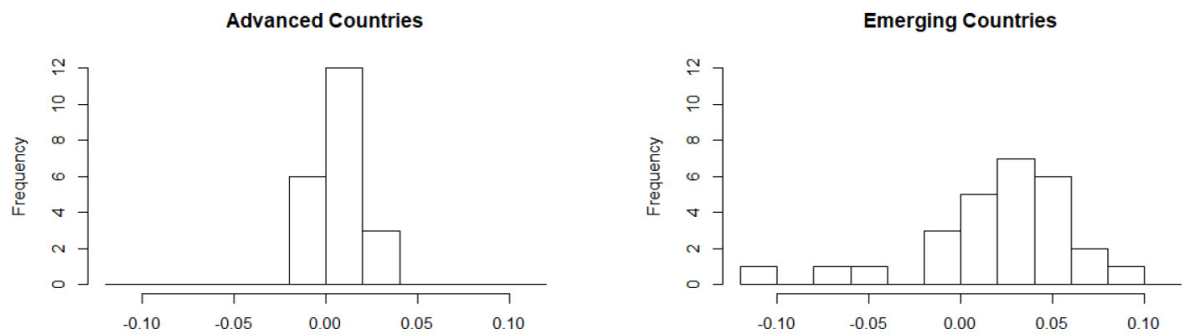

Model 3
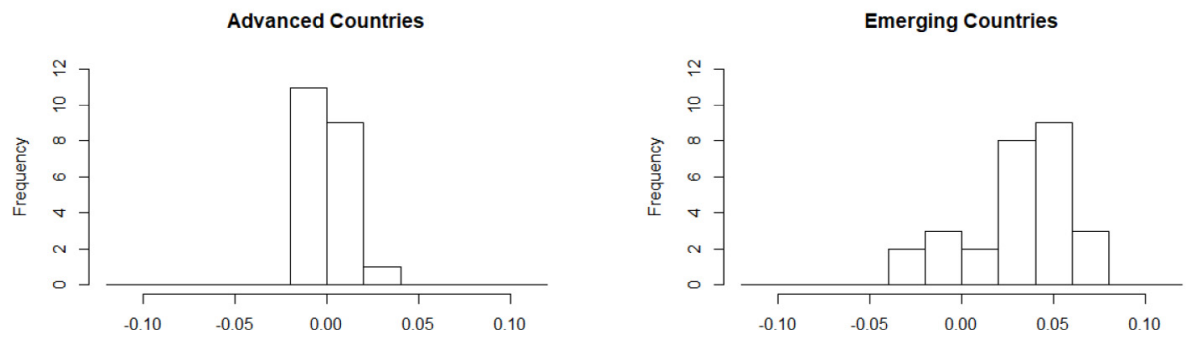

Fig. 1. Differences in forecast accuracy. Notes: Histograms for country-level differences in accuracy (expert forecast RMSEs minus model forecast RMSEs). A positive number indicates that expert forecasts are less accurate.

\subsection{Excessive optimism}

Earlier analyses of GDP growth forecasts have documented excessive optimism. In particular, this pattern has been detected in more distant forecast targets (De Resende, 2014; Frankel, 2011; Ho \& Mauro, 2016). This subsection verifies this property by studying the forecast errors from experts and Model 3.

Table 5 reports descriptive statistics and the associated tests. It is observed that the mean errors in the case of expert forecasts are negative and significantly different from zero. That is, the forecasts are too optimistic on average. This property is observed for both advanced and emerging countries. The estimated unconditional bias in the case of emerging countries is -0.058 , whereas that in the case of advanced economies is almost $30 \%$ smaller, though still statistically and economically significant. In contrast, no significant unconditional bias is observed in the case of Model 3 forecasts. For emerging economies, this evidence serves as a first explanation for differences in forecast accuracy. In the case of advanced economies, this evidence serves as a further indication that, while experts were not found to underperform models, there might be space for improvements in forecasts.

\subsection{Conditional biases}

In addition to unconditional biases, the relative forecast performances could also be explained by differential responses to changing economic conditions. Prior contributions in macroeconomic contexts provide evidence and theoretical frameworks that suggest the plausibility 
Model 1
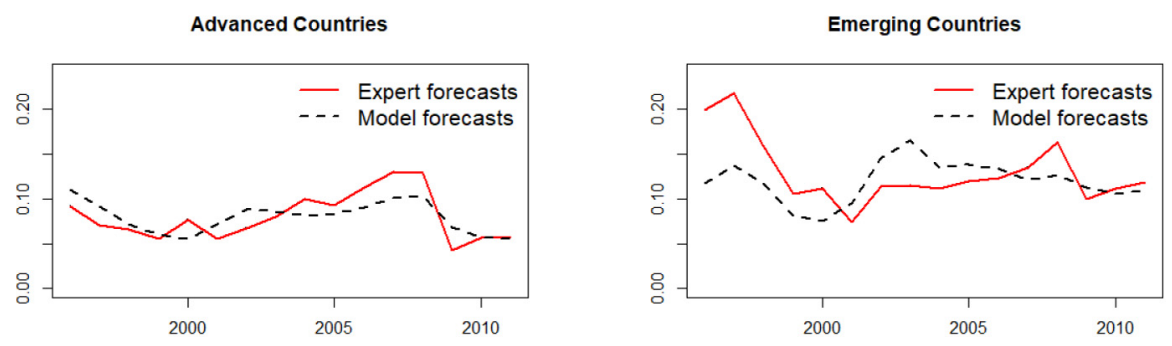

Model 2
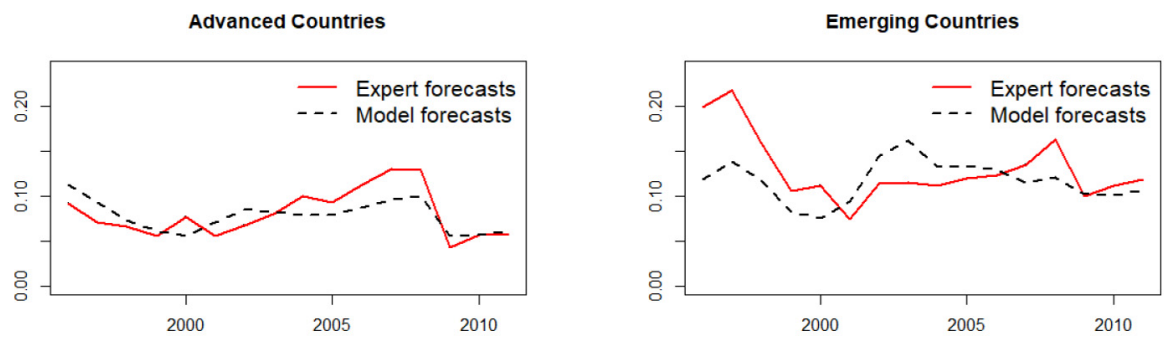

Model 3
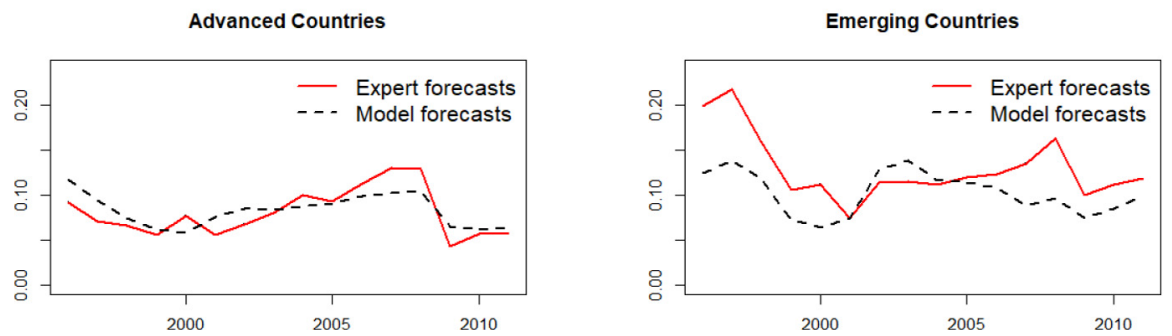

Fig. 2. Differences in forecast accuracy. Notes: RMSEs of expert and model forecasts by year of forecast release.

of inefficient responses. A body of literature linked to the idea of inattention has developed models and empirical analyses in which economic actors fail to incorporate all available information. ${ }^{10}$ According to these contributions, limited cognitive resources can lead to expectations that underreact to available information. According to this perspective, inefficiencies in growth forecasts could be associated with some form of inattention. For example, economic forecasts could underreact to information linked to the current account balance. From a different but complementary perspective, some authors have identified inefficiencies associated with an excessive extrapolation of recent trajectories, or, in other words, a disregard for

\footnotetext{
10 See for example Coibion and Gorodnichenko (2015), Sims (2003) and Woodford (2009).
}

mean reversal in stochastic processes. ${ }^{11}$ This type of inefficiency can result in economic forecasts that overreact to the available information. For example, forecasts could manifest excessive reactions to accelerations in GDP growth.

While a detailed evaluation of the nature of the expectation formation process is beyond the scope of the current work, the arguments described above serve as an indication of the plausibility of inefficient responses. The presence of inefficiencies is to be evaluated empirically. A model that estimates conditional mean forecast errors is used with this objective. This evaluation is later complemented with a second analysis of differential reactions in expert and model forecasts.

11 See Barberis, Greenwood, Jin, and Shleifer (2015), Beshears, Choi, Fuster, Laibson, and Madrian (2013), and Greenwood and Shleifer (2014). 
Table 5

Mean forecast errors.

\begin{tabular}{lll}
\hline & $\begin{array}{l}\text { Expert forecasts } \\
{[\mathrm{A}]}\end{array}$ & $\begin{array}{l}\text { Model 3 forecasts } \\
{[\mathrm{B}]}\end{array}$ \\
\hline $\begin{array}{l}\text { All countries } \\
\text { Mean forecast error }\end{array}$ & -0.051 & -0.007 \\
$t$-statistic & -4.83 & -0.37 \\
$p$-value & 0.000 & 0.709 \\
$\begin{array}{l}\text { Advanced countries } \\
\text { Mean forecast error }\end{array}$ & -0.041 & -0.004 \\
$t$-statistic & -3.64 & -0.27 \\
$p$-value & 0.000 & 0.791 \\
$\begin{array}{l}\text { Emerging countries } \\
\text { Mean forecast error }\end{array}$ & -0.058 & -0.011 \\
$t$-statistic & -3.30 & -0.71 \\
$p$-value & 0.001 & 0.474 \\
\hline
\end{tabular}

Notes: The $t$-statistics are computed using year and country clusters following Cameron et al. (2011) and a subset of forecasts with non-overlapping target windows.

Under efficient forecasts that minimize square errors, the conditional mean forecast errors are zero. If the forecasts overreact or underreact to incoming information, the conditional mean forecast errors will vary with changes in the information set. More formally, the evaluation is carried out by estimating a linear model. Let $f e_{c[t+1, t+5]}$ denote forecast errors; that is, the difference between actual growth and the expert forecast. Then, conditional biases can be measured by estimating the following empirical model:

$f e_{c[t+1, t+5]}=\alpha_{c}+\alpha_{t}+\sum_{i=1}^{N} \beta_{i} x_{c t}^{i}+u_{c, t}$,

where $\alpha_{c}$ is a country fixed effect, $\alpha_{t}$ is a time fixed effect and $x_{c t}^{i}$, with $i \in 1, \ldots, N$, represents a variable with respect to which conditional biases are assessed. The estimated coefficient $\beta_{i}$ captures the presence of conditional biases associated with indicator $x_{c t}^{i}$. Thus, a coefficient that is different from zero is consistent with a scenario in which the forecasts suffer conditional biases. The responses to two relevant aspects are considered: growth accelerations and the current account balance. The growth accelerations for country $c$ in year $t$ are given by the cumulative growth in the previous five-year period ( $[t-$ $5, t-1])$ minus the average cumulative rate for the two immediately preceding (and non-overlapping) five-year periods $([t-10, t-6]$ and $[t-15, t-11])$. Current account information corresponds to the balance in the year before forecasts are released. According to the description provided in the data section, the information on growth acceleration and the current account balance was available at the time of forecast release.

Table 6 reports the evidence on the conditional mean errors of expert forecasts. Column 1 shows that conditional biases are detected when growth accelerations are considered. Consistent with overreactions, more intense accelerations are associated with smaller mean forecast errors. That is, the mean expert forecasts increase more than the mean realized growth. According to the estimated parameter, an increment of $1 \%$ in the acceleration metric is associated with a $0.22 \%$ drop in the subsequent mean forecast error. A flexible model that allows for different coefficients depending on whether an economy is classified as advanced or emerging is also estimated. The estimation, reported in column 3 , indicates that there is no significant difference in the association. In other words, conditional biases of similar intensities are documented in the cases of advanced and emerging economies.

The estimation of a model that includes the current account balance as an explanatory variable points to a different type of inefficiency. The estimated coefficient reported in column 2 is significant and positive, which is consistent with underreactions. An increment in the current account balance is associated with an increment in the mean growth realization that is larger than the change in the mean growth forecast. In this case, the estimation of the flexible model indicates that the inefficiency is detected only in the case of emerging countries. For this second group of economies, the estimated parameter reported in column 4 indicates that an increment of $1 \%$ in the current account balance in the previous year is associated with an increment of $1.05 \%$ in the mean forecast error over the next five-year window.

As was indicated in the introduction, the systematic errors reported above could be an inevitable feature in contexts with structural breaks, and one that is explained by learning processes. This is why it is of interest to evaluate whether expert forecasts and model forecasts differ in terms of their responses to growth accelerations and the current account balance. Equal responses would lend support to the idea that the conditional biases documented earlier are explained by learning. On the other hand, differential responses could suggest inefficient reactions in expert forecasts. Heterogeneities in responses are

Table 6

Conditional mean forecast errors.

\begin{tabular}{lllllll}
\hline & {$[1]$} & {$[2]$} & {$[3]$} & {$[4]$} & {$[5]$} & {$[6]$} \\
\hline Growth accel. & $-0.224^{* * * *}$ & - & $-0.189^{* * * *}$ & - & $-0.184^{* * *}$ & $-0.188^{* * * *}$ \\
& $(0.068)$ & & $(0.098)$ & & $(0.063)$ & $(0.060)$ \\
Gr. accel. $\times$ Emerg. & - & - & -0.046 & - & - & 0.053 \\
& & & $(0.098)$ & & & $(0.059)$ \\
Current account bal. & - & $0.587^{* * *}$ & - & 0.089 & $0.499^{* * *}$ & 0.076 \\
& & $(0.120)$ & & $(0.076)$ & $(0.072)$ & $(0.062)$ \\
C. A. bal. $\times$ Emerg. & - & - & - & $1.055^{* * *}$ & - & $0.972^{* * *}$ \\
& & & & $(0.319)$ & & $(0.147)$ \\
\hline
\end{tabular}

Notes: The table shows the estimated coefficients $(\beta \mathrm{s})$ for linear models of forecast errors. The $t$-statistics are computed using the Driscoll and Kraay (1998) robust covariance matrix estimator. ${ }^{*},{ }^{* * *}$ and ${ }^{* * *}$ indicate significance at the $10 \%, 5 \%$, and $1 \%$ levels, respectively. 
Table 7

Differences in reaction to information.

\begin{tabular}{|c|c|c|c|c|c|c|}
\hline & [1] & [2] & [3] & [4] & [5] & [6] \\
\hline Growth accel. & $\begin{array}{l}0.083^{* * * *} \\
(0.032)\end{array}$ & - & $\begin{array}{l}0.049 \\
(0.035)\end{array}$ & - & $\begin{array}{l}0.073^{* * * *} \\
(0.026)\end{array}$ & $\begin{array}{l}0.036 \\
(0.035)\end{array}$ \\
\hline Gr. accel. × Emerg. & - & - & $\begin{array}{l}0.046 \\
(0.062)\end{array}$ & - & - & $\begin{array}{l}-0.002 \\
(0.050)\end{array}$ \\
\hline Current account bal. & - & $\begin{array}{l}-0.234^{* * *} \\
(0.081)\end{array}$ & - & $\begin{array}{l}0.243^{* * * *} \\
(0.046)\end{array}$ & $\begin{array}{l}-0.207^{* * *} \\
(0.065)\end{array}$ & $\begin{array}{l}0.234^{* *} \\
(0.045)\end{array}$ \\
\hline C. A. bal. $\times$ Emerg. & - & - & - & $\begin{array}{l}-0.963^{* * * *} \\
(0.080)\end{array}$ & - & $\begin{array}{l}-0.921^{* * *} \\
(0.073)\end{array}$ \\
\hline
\end{tabular}

Note: $t$-statistics are computed using the Driscoll and Kraay (1998) robust covariance matrix estimator. ${ }^{*},{ }^{* *}$ and ${ }^{* * *}$ indicate significance at the $10 \%, 5 \%$, and $1 \%$ levels, respectively.

evaluated formally through a simple empirical model. Let $d f_{c[t+1, t+5]}$ be the difference between the expert forecast and the Model 3 forecast. Then, heterogeneous responses can be measured by estimating the following empirical model:

$d f_{c[t+1, t+5]}=\alpha_{c}+\alpha_{t}+\sum_{i=1}^{N} \beta_{i} x_{c t}^{i}+u_{c, t}$,

where $\alpha_{c}$ is a country fixed effect, $\alpha_{t}$ is a time fixed effect and $x_{c t}^{i}$ represents a variable that captures the relevant conditions that can be observed at the time at which the forecasts are generated. The estimated coefficient $\beta_{i}$ would capture any systematic difference in the response to changes in $x_{c t}^{i}$. As in the previous exercise, two variables are considered: growth accelerations and the current account balance.

Table 7 shows the estimated differential responses for these two indicators. Columns 1 and 2 show that, considered independently, the forecasts respond differently to changes in the two variables used in the analysis. In the case of growth accelerations, the estimations indicate that the expert forecasts increase more in response. On the other hand, the model forecasts increase more with an increment in the current account balance. These statistically significant differential responses provide support for the interpretation of conditional biases as manifestations of inefficient responses to incoming economic information. Even when the possibility of structural breaks and the associated learning is taken into account, expert forecast seem to overreact to growth accelerations and underreact to information provided by the current account balance.

When models with different slopes for emerging and advanced countries are estimated, the evidence suggests that the differential responses are concentrated in the case of emerging countries. In particular, column 4 shows that the estimated response is approximately three times larger in absolute value in the case of emerging economies. These heterogeneous responses are consistent with the relative performances of expert and model forecasts documented in the previous sections.

\subsection{Performance conditional on growth realizations}

The analysis reported above indicates that expert forecasts are characterized by excessive optimism and excessive reactions to growth accelerations. These properties suggest that the performances of expert forecasts will differ significantly depending on growth realizations. This subsection provides a detailed analysis of relative performances conditional on realizations. It must be noted that the decomposition is not intended for identifying inefficiencies, but is a complementary quantitative description of the forecasts' properties.

We obtained this description by dividing the sample into thirds according to how the realized growth in the target period compared to that observed in the five-year period preceding the forecast release. Thus, periods of growth acceleration correspond to the third of the sample with the highest difference in cumulative growth in those two periods. Periods of growth deceleration are associated with the third of the sample with the lowest values for the difference, while periods of moderate change in growth rates are associated with intermediate values.

Table 8 shows the decomposition described earlier. As expected, independently of the type of forecast, growth realizations that are close to the levels observed in the previous periods are associated with the highest levels of accuracy. On the other hand, while the model forecasts' RMSE conditional on accelerations is very close to their RMSE conditional on decelerations, the expert forecasts' RMSE is notoriously larger in the case of decelerations. This implies that the RMSE of expert forecasts rises to 0.159 for one-third of the sample, namely during times of deceleration. In the case of emerging economies, the

Table 8

Accuracy and growth outcomes.

\begin{tabular}{|c|c|c|c|}
\hline & All & $\begin{array}{l}\text { Adv. } \\
\text { countries }\end{array}$ & $\begin{array}{l}\text { Emerg. } \\
\text { countries }\end{array}$ \\
\hline \multicolumn{4}{|c|}{ Growth accelerations: } \\
\hline A. Expert RMSE & 0.092 & 0.072 & 0.102 \\
\hline B. Model RMSE & 0.110 & 0.099 & 0.119 \\
\hline Ratio (B/A) & 1.198 & 1.382 & 1.162 \\
\hline \multicolumn{4}{|c|}{$\begin{array}{l}\text { Moderate change on } \\
\text { growth rates: }\end{array}$} \\
\hline A. Expert RMSE & 0.074 & 0.058 & 0.097 \\
\hline B. Model RMSE & 0.058 & 0.054 & 0.063 \\
\hline Ratio (B/A) & 0.782 & 0.929 & 0.652 \\
\hline \multicolumn{4}{|c|}{ Growth decelerations: } \\
\hline A. Expert RMSE & 0.159 & 0.140 & 0.185 \\
\hline B. Model RMSE & 0.111 & 0.099 & 0.122 \\
\hline Ratio (B/A) & 0.697 & 0.828 & 0.658 \\
\hline
\end{tabular}

Notes: The forecasting sample was divided using terciles for the difference between growth realization and growth during the five-year period preceding the forecast release $\left(g_{c[t-1, t+5]}-g_{c[t-5, t-1]}\right)$. 
RMSE of the expert forecasts during times of deceleration is 0.185 , that is, $81 \%$ higher than that observed during accelerations and 51\% higher than that of the model forecasts for the same set of prediction tasks.

In contrast, expert forecasts perform better than model forecasts during periods of accelerations. This relative performance is observed for both country groups, though with different intensities. In the case of advanced countries, conditional on accelerations, the model forecasts' RMSE is 38\% larger than the expert forecasts' RMSE. In the case of emerging countries, the model forecasts' RMSE is $16 \%$ larger than the expert forecasts' RMSE. While these differences are expected given previous findings, they allow for more precise characterizations of the forecasts' properties.

This section has explored various regularities that can explain and illustrate differences in performance. In addition to the forecast evaluations presented in the previous sections, the analyses presented in the current section provide specific insights into some characteristics of expert forecasts that can explain their lower level of accuracy compared to simple model forecasts. A straightforward set of recommendations emerges from these findings. First, the expert forecast performance would benefit from a reduction in the level of optimism for both groups of economies. Second, more attention should be placed on the information carried by the current account balance. This seems to be especially important for emerging economies. Finally, a third recommendation is associated with the extrapolation of recent growth outcomes. The evidence suggests that the level of persistence in growth trajectories is lower than that manifested by expert forecasts. A more agnostic view of the information provided by the recent growth performance could result in more precise forecasts. The evidence suggests that this observation is valid for both country groups.

\section{Conclusions}

This study evaluates the medium-term GDP growth forecasts released by experts. The evaluation involves comparing the performances of expert forecasts with those of forecasts generated by simple models trained on historical data.

The results indicate that no noticeable difference in performance is observed for advanced economies. In contrast, a significant difference in accuracy is observed in the case of emerging economies. In this case, the model forecasts' RMSEs are between $9 \%$ and $22 \%$ lower than the expert forecasts' RMSEs. These differences in performance are also observed under alternative forecasting methodologies and for an alternative set of expert forecasts. Thus, the simple models used in this study are unable to perform better than experts in short-term prediction tasks, indicating that medium- and short-horizon forecasts display different traits.

Further exercises have identified properties of expert forecasts that can explain their relative performances, leading to specific recommendations for forecasting practices. It has been shown that the differences in performance can be linked to the presence of optimism bias, excessive responses to growth accelerations and insufficient reactions to the current account balance. These features are not present only in the case of forecasts for emerging countries, but are more visible in this case.

The forecasting models used in this work are notably simple. Future research could evaluate the relative performances of models that exploit larger data sets or that allow for nonlinearities. Also, while some explanations for the reported differences in performance have been identified, future contributions could evaluate the existence of complementary explanations. This study has focused on medium-term GDP growth predictions, but a natural extension could consider similar exercises associated with other macroeconomic variables. Given the non-trivial interactions among macroeconomic indicators, this extension could complement the current study. More specifically, correlations in forecast errors across different variables might allow for a more precise understanding of the properties that characterize macroeconomic forecasts.

\section{Appendix 6. Supplementary data}

Supplementary material related to this article can be found online at https://doi.org/10.1016/j.ijforecast.2019. 03.004 .

\section{Appendix A. List of sampled countries}

\section{Advanced:}

Australia, Austria, Belgium, Canada, Denmark, Finland, France, Germany, Ireland, Italy, Japan, Saudi Arabia, Netherlands, New Zealand, Norway, Singapore, Spain, Sweden, United Arab Emirates, United Kingdom, and United States.

\section{Emerging:}

Argentina, Bolivia, Brazil, Chile, China, Colombia, Czech Republic, Ecuador, Egypt, Greece, Hungary, India, Indonesia, Malaysia, Mexico, Pakistan, Peru, Philippines, Poland, Portugal, Russia, South Africa, South Korea, Thailand, Turkey, Uruguay and Vietnam.

\section{Appendix B. RMSEs by country}




\begin{tabular}{|c|c|c|c|}
\hline \multicolumn{4}{|c|}{ Advanced countries } \\
\hline & $\begin{array}{l}\text { Model } 3 \\
{[\mathrm{~A}]}\end{array}$ & $\begin{array}{l}\text { Expert } \\
{[B]}\end{array}$ & $\begin{array}{l}\text { Ratio } \\
{[\mathrm{A}] /[\mathrm{B}]}\end{array}$ \\
\hline Australia & 0.037 & 0.043 & 0.873 \\
\hline Austria & 0.052 & 0.052 & 1.013 \\
\hline Belgium & 0.060 & 0.052 & 1.159 \\
\hline Canada & 0.052 & 0.055 & 0.943 \\
\hline Denmark & 0.061 & 0.072 & 0.846 \\
\hline Finland & 0.089 & 0.079 & 1.131 \\
\hline France & 0.069 & 0.073 & 0.944 \\
\hline Germany & 0.050 & 0.065 & 0.765 \\
\hline Ireland & 0.174 & 0.179 & 0.971 \\
\hline Italy & 0.121 & 0.102 & 1.192 \\
\hline Japan & 0.071 & 0.086 & 0827 \\
\hline Netherlands & 0.072 & 0.077 & 0.931 \\
\hline New Zealand & 0.069 & 0.067 & 1.028 \\
\hline Norway & 0.040 & 0.033 & 1.204 \\
\hline Saudi Arabia & 0.050 & 0.074 & 0.674 \\
\hline Singapore & 0.105 & 0.100 & 1.045 \\
\hline Spain & 0.104 & 0.096 & 1.087 \\
\hline Sweden & 0.050 & 0.042 & 1.194 \\
\hline United Arab & 0.156 & 0.143 & 1.092 \\
\hline Emirates & & & \\
\hline United Kingdom & 0.065 & 0.078 & 0.828 \\
\hline United States & 0.071 & 0.070 & 1.023 \\
\hline
\end{tabular}

\section{RMSEs by country:}

\begin{tabular}{llll}
\hline Emerging countries & \multicolumn{3}{l}{$l$} \\
\hline & $\begin{array}{l}\text { Expert } \\
\text { forecasts }\end{array}$ & Model 3 & Difference \\
& {$[\mathrm{A}]$} & {$[\mathrm{B}]$} & {$[\mathrm{A}]-[\mathrm{B}]$} \\
\hline Argentina & 0.188 & 0.222 & 0.847 \\
Bolivia & 0.054 & 0.110 & 0.492 \\
Brazil & 0.097 & 0.123 & 0.790 \\
Chile & 0.055 & 0.127 & 0.437 \\
China & 0.149 & 0.120 & 1.247 \\
Colombia & 0.094 & 0.113 & 0.827 \\
Czech Republic & 0.112 & 0.158 & 0.708 \\
Ecuador & 0.077 & 0.114 & 0.677 \\
Egypt & 0.083 & 0.126 & 0.659 \\
Greece & 0.135 & 0.185 & 0.770 \\
Hungary & 0.102 & 0.139 & 0.736 \\
India & 0.116 & 0.096 & 1.214 \\
Indonesia & 0.127 & 0.167 & 0.761 \\
South Korea & 0.048 & 0.103 & 0.463 \\
Malaysia & 0.083 & 0.141 & 0.587 \\
Mexico & 0.070 & 0.123 & 0.570 \\
Pakistan & 0.053 & 0.105 & 0.502 \\
Peru & 0.110 & 0.137 & 0.802 \\
Philippines & 0.034 & 0.109 & 0.310 \\
Poland & 0.056 & 0.091 & 0.614 \\
Portugal & 0.054 & 0.122 & 0.445 \\
Russia & 0.190 & 0.204 & 0.933 \\
South Africa & 0.058 & 0.094 & 0.613 \\
Thailand & 0.123 & 0.172 & 0.716 \\
Turkey & 0.104 & 0.102 & 1.022 \\
Uruguay & 0.196 & 0.191 & 1.023 \\
Vietnam & 0.094 & 0.075 & 1.258 \\
\hline & & &
\end{tabular}

\section{Appendix C. Robustness tests}

This appendix presents a series of alternative exercises. The variations involve alternative forecast model specifications, the analysis of different data, and changes in the prediction task. These extensions shed further light on the findings reported above regarding the relative forecasting accuracy.

The medium-term forecasts reported in the main text were generated by estimating five-year-ahead prediction models using data from a country panel. A natural question is whether the use of alternative model specifications would result in a better forecasting performance. One alternative involves estimating a one-year-ahead prediction model and computing medium-term forecasts iterating over that model. In another alternative, predictions are generated using individual country time series models rather than data from multiple countries. These two alternatives are evaluated below.

In addition to alternative model specifications, the findings on forecast accuracy are evaluated by considering alternative forecasting horizons and a different source of expert forecasts. The relative performances of experts versus simple models are evaluated in short-horizon prediction tasks, with current-year and one-year-ahead forecast horizons being considered. Finally, the stability of the model parameters is evaluated, with the estimated parameters using data for the period 1961-1996 being reported.

\section{C.1. Iterative forecasts}

The forecasting exercise reported earlier involved simple models being trained to generate direct predictions for a five-year horizon. An alternative specification involves training one-year-ahead models and iterating over this model to generate predictions for the subsequent years. As was indicated by Marcellino, Stock, and Watson (2006), the preferred specification remains an empirical matter. This subsection evaluates the performance of the iterative alternative.

In the spirit of Corsi (2009), one-year-ahead forecasts are generated using heterogeneous autoregressive (HAR) models. A second equation is introduced for generating forecasts for the GDP per capita. Keeping the model as simple as possible, the same set of predictors is used in this second equation. Model 1-I is given by the following equations:

$$
\begin{aligned}
g_{c, t+1}= & \alpha^{1}+\sum_{k \in\{1,5,15\}} \beta_{k}^{1} g_{c[t-k, t-1]} \\
+ & \beta_{g d p p c}^{1} g d p p c_{c, t-1}+u_{c, t-1}^{1} \\
g d p p c_{c, t}= & \alpha^{2}+\sum_{k \in\{1,5,15\}} \beta_{k}^{2} g_{c[t-k, t-1]} \\
& +\beta_{g d p p c}^{2} g d p p c_{c, t-1}+u_{c, t-1}^{2} .
\end{aligned}
$$

As in the case of direct forecasts, a second model (Model 2-I) incorporates the current account balance as a predictor. This modification requires the introduction of an additional equation to generate forecasts of current account 
balances:

$$
\begin{aligned}
g_{c, t+1}= & \alpha^{1}+\sum_{k \in\{1,5,15\}} \beta_{k}^{1} g_{c[t-k, t-1]}+\beta_{g d p p c}^{1} g d p p c_{c, t-1} \\
& +\beta_{c a b}^{1} c a b_{c, t-1}+u_{c, t-1}^{1}
\end{aligned}
$$$$
g d p p c_{c, t}=\alpha^{2}+\sum_{k \in\{1,5,15\}} \beta_{k}^{2} g_{c[t-k, t-1]}+\beta_{g d p p c}^{2} g d p p c_{c, t-1}
$$$$
+\beta_{c a b}^{2} c a b_{c, t-1}+u_{c, t-1}^{2}
$$

$$
\begin{aligned}
c a b_{c, t}= & \alpha^{3}+\sum_{k \in\{1,5,15\}} \beta_{k}^{3} g_{c[t-k, t-1]}+\beta_{g d p p c}^{3} g d p p c_{c, t-1} \\
& +\beta_{c a b}^{3} c a b_{c, t-1}+u_{c, t-1}^{3} .
\end{aligned}
$$

Also, replicating the previously-reported exercises, we estimate a third model (Model 3-I) with two sets of forecasting equations: one set for advanced economies and another for emerging economies.

For each year $t$ in the set $\{1996, \ldots, 2011\}$, we generate five-year-ahead forecasts using the models trained for one-year-ahead forecasts. In the first step, one-yearahead forecasts are generated using the available information for the predictors. These forecasts are then used to compute expected values for the predictors in the following year and the model is used again, this time to generate two-year-ahead forecasts. This procedure is repeated three more times to generate forecasts for the remaining years in the prediction target window. In the last step, yearly forecasts for GDP growth are combined in order to compute cumulative growth forecasts.

The results associated with these forecasting models are shown in Table C.1, and are similar to those observed in the original exercise. The model forecasts' RMSEs are lower in the case of advanced economies, but no significant difference in accuracy is observed according to the tests for differences. In the case of emerging economies, the model forecasts outperform the expert forecasts. In this case, the RMSEs are between $12 \%$ and $21 \%$ lower, depending on the model specification. Replicating the relative performances observed in the previous section, the highest accuracy is observed for Model 3-I, while improvements in performance are observed when Models 1-I and 2-I are used instead of direct forecasts under Models 1 and 2, respectively. These improvements are reflected in both the accuracy metric and the $p$-values of the tests for differences. In the case of Model 3-I and emerging economies, its forecasting performance is slightly worse than that observed in the case of Model 3.

In summary, the performances of models under direct and iterative forecasting are very similar. That is, there is no strong evidence suggesting that one method should be preferred over the other. At the same time, the computation of iterative forecast serves as a robustness test of the findings reported with direct forecasts.

\section{C.2. Forecasts from time series models}

Another variation in the forecast model involves the implementation of individual country time series regressions. This subsection trains the equations used in the
Table C.1

Comparison of accuracy levels: iterative forecasts.

\begin{tabular}{llll}
\hline & All & $\begin{array}{l}\text { Adv. } \\
\text { countries }\end{array}$ & $\begin{array}{l}\text { Emerg. } \\
\text { countries }\end{array}$ \\
\hline A. Expert forecasts & 0.114 & 0.085 & 0.134 \\
RMSE & & & \\
B. Model 1-I & 0.103 & 0.079 & 0.118 \\
RMSE & 0.896 & 0.939 & 0.882 \\
Ratio (B/A) & -2.02 & -0.44 & -1.97 \\
$t$-statistic & 0.043 & 0.661 & 0.048 \\
$p$-value & & & \\
C. Model 2-I & 0.098 & 0.080 & 0.111 \\
RMSE & 0.856 & 0.949 & 0.822 \\
Ratio (C/A) & -2.27 & -0.12 & -2.36 \\
$t$-statistic & 0.023 & 0.908 & 0.019 \\
$p$-value & & & \\
D. Model 3-I & 0.095 & 0.079 & 0.106 \\
RMSE & 0.828 & 0.932 & 0.791 \\
Ratio (D/A) & -2.43 & -0.32 & -2.43 \\
$t$-statistic & 0.015 & 0.752 & 0.015 \\
$p$-value & & & \\
\hline
\end{tabular}

Notes: The table shows $t$-statistics and $p$-values from two-tailed tests for forecast accuracy differences as proposed by Pesaran et al. (2009). The tests are computed using a subsample of forecasts for non-overlapping five-year windows. Forecast combination involves the equal-weighted average of the expert forecast and the Model 3 forecast.

Table C.2

Comparison of accuracy levels: individual country time series regressions.

\begin{tabular}{llll}
\hline & All & $\begin{array}{l}\text { Adv. } \\
\text { countries }\end{array}$ & $\begin{array}{l}\text { Emerg. } \\
\text { countries }\end{array}$ \\
\hline RMSEs: & & & \\
$\begin{array}{l}\text { A. Expert forecasts } \\
\begin{array}{l}\text { B. Time series model } \\
\text { forecasts }\end{array}\end{array}$ & 0.106 & 0.088 & 0.120 \\
Ratio (B/A) & 1.375 & 0.092 & 0.180 \\
\hline
\end{tabular}

original exercise with a more restrictive information set. More specifically, we use the equation used in Models 2 and 3 , the specification with two additional predictors. However, instead of training the model using data from multiple countries, the models are trained using exclusively data from the country for which the GDP growth is to be predicted. We ensure that enough data is used to train the model by generating and evaluating forecasts only if at least 20 observations are available for training the model.

The results in Table C.2 indicate that forecasts from individual country time series regressions are unable to perform better than expert forecasts. In the case of emerging economies, this negative result is in sharp contrast to that observed when models are trained using pooled data from multiple countries. In other words, the positive results reported in the previous section are based, to an important extent, on the ability to learn from past growth trajectories observed in multiple countries. Medium-term forecasts tend to suffer from the problem of a limited number of available observations, and learning about other countries seems to be especially valuable as a result. 
Table C.3

Comparison of accuracy levels.

\begin{tabular}{llll}
\hline & All & $\begin{array}{l}\text { Adv. } \\
\text { countries }\end{array}$ & $\begin{array}{l}\text { Emerg. } \\
\text { countries }\end{array}$ \\
\hline $\begin{array}{l}\text { Forecast horizon: current } \\
\text { year }\end{array}$ & & & \\
A. Expert RMSE & 0.018 & 0.016 & 0.020 \\
$\begin{array}{l}\text { B. Model RMSE } \\
\text { Ratio (B/A) }\end{array}$ & 0.031 & 0.025 & 0.035 \\
$t$-statistic & 1.665 & 1.531 & 1.736 \\
$p$-value & 3.78 & 2.11 & 3.32 \\
Forecast horizon: & 0.000 & 0.034 & 0.001 \\
one-year-ahead & & & \\
A. Expert RMSE & 0.032 & 0.026 & 0.036 \\
B. Model RMSE & 0.030 & 0.026 & 0.034 \\
Ratio (B/A) & 0.946 & 0.986 & 0.929 \\
$t$-statistic & 0.73 & 0.47 & 1.09 \\
$p$-value & 0.460 & 0.632 & 0.276 \\
& & & \\
\hline
\end{tabular}

Notes: The table shows $t$-statistics and $p$-values from two-tailed tests of forecast accuracy differences as proposed by Pesaran et al. (2009).

\section{C.3. Short-horizon forecasts}

Can simple models outperform experts at different prediction tasks? In particular, what are their relative performances in the case of short-term forecasts? This subsection evaluates short-term forecasts, and, more specifically, the relative performances of current-year and one-year-ahead forecasts are evaluated. The simple HAR models that were used to generate iterative forecasts are trained to generate short-term forecasts. The model is given by:

$$
\begin{aligned}
g_{c, t+h}= & \alpha^{1}+\sum_{k \in\{1,5,15\}} \beta_{k}^{1} g_{c[t-k, t-1]} \\
& +\beta_{g d p p c}^{1} g d p p c_{c, t-1}+u_{c, t-1}^{1},
\end{aligned}
$$

where $h \in\{0,1\}$ indicates the time horizon of the prediction task. Table C.3 reports information on the performances of experts and models on these short-horizon prediction tasks. In the case of current-year forecasts, experts are found to outperform the models significantly, for both advanced and emerging economies. When one-yearahead forecasts are considered, no statistically significant difference in performances is found, though the computed RMSEs are lower in the case of model forecasts for both country groups.

It is worth noting that while the expert forecast accuracy drops sharply with a one-year increment in forecast horizon, no similar change is observed in the case of model forecast accuracy. This contrast suggests that the simple models proposed in this exercise have a hard time capturing the short-term information that is incorporated by experts.

\section{C.4. Parameter stability}

We evaluate the parameter stability by showing in Table C.4 the estimated forecasting models that were used to predict growth for the year 1996. These fitted models correspond to the first set of forecasting tasks,
Table C.4

Estimated forecasting models: 1961-1996.

\begin{tabular}{lllll}
\hline & {$[1]$} & {$[2]$} & [3: Adv.] & [3: Em.] \\
\hline$\alpha$ & $0.152^{* * *}$ & $0.149^{* * *}$ & $0.224^{* *}$ & $0.219^{* * *}$ \\
& $(3.4)$ & $(3.4)$ & $(2.6)$ & $(3.5)$ \\
$\beta_{t-5, t-1}$ & $0.148^{* * * *}$ & $0.151^{* *}$ & 0.149 & $0.151^{* *}$ \\
& $(2.3)$ & $(2.3)$ & $(1.1)$ & $(2.5)$ \\
$\beta_{t-10, t-6}$ & 0.074 & 0.078 & -0.033 & 0.125 \\
& $(0.7)$ & $(0.8)$ & $(-0.2)$ & $(1.5)$ \\
$\beta_{t-15, t-11}$ & 0.069 & 0.068 & 0.122 & 0.091 \\
& $(1.1)$ & $(1.1)$ & $(1.1)$ & $(0.7)$ \\
$\beta_{g d p p c}$ & $-0.126^{* * *}$ & $-0.122^{* * *}$ & $-0.201^{*}$ & $-0.538^{* * *}$ \\
& $(-2.6)$ & $(-2.6)$ & $(-1.7)$ & $(-3.9)$ \\
$\beta_{\text {cab }}$ & - & -0.082 & $-0.170^{* * *}$ & 0.816 \\
& & $(-0.4)$ & $(-3.5)$ & $(1.4)$ \\
$N$ & 615 & 615 & 300 & 315 \\
$R^{2}$ & 0.181 & 0.182 & 0.203 & 0.22
\end{tabular}

Notes: The estimated coefficients correspond to the models used to generate predictions in the year 1996. The $t$-statistics (in parentheses) were computed using year and country clusters following Cameron et al. (2011). ", "** and ${ }^{* * *}$ indicate significance at the $10 \%, 5 \%$, and $1 \%$ levels, respectively.

and were estimated using a significantly smaller number of observations. Overall, the estimated associations seem quite stable. Positive but weak associations with lagged growth outcomes are present in both set of estimations. In the case of GDP per capita, the significant negative association is also stable. One exception is the case of the current account balance: while the positive association remains strong in the case of emerging economies, the estimations for the early sample point to a negative association between current account balance and expected growth in the case of advanced economies.

\section{References}

Ager, P., Kappler, M., \& Osterloh, S. (2009). The accuracy and efficiency of the Consensus Forecasts: A further application and extension of the pooled approach. International Journal of Forecasting, 25(1), $167-181$

Aromí, J. D. (2018). GDP growth forecasts and information flows: is there evidence of overreaction? International Finance, 21(2), 122-139.

Barberis, N., Greenwood, R., Jin, L., \& Shleifer, A. (2015). X-CAPM: An extrapolative capital asset pricing model. Journal of Financial Economic, 115(1), 1-24.

Barro, R. J. (1991). Economic growth in a cross section of countries. Quarterly Journal of Economics, 106(2), 407-443.

Batchelor, R. (2007). Bias in macroeconomic forecasts. International Journal of Forecasting, 23(2), 189-203.

Beshears, J., Choi, J. J., Fuster, A., Laibson, D., \& Madrian, B. C. (2013). What goes up must come down? Experimental evidence on intuitive forecasting. American Economic Review, 103(3), 570-574.

Blanchard, O., \& Giavazzi, F. (2002). Current account deficits in the euro area: the end of the Feldstein-Horioka puzzle? Brookings Papers on Economic Activity, 2002(2), 147-209.

Calvo, G. A. (1998). Capital flows and capital market crises: the simple economics of sudden stops. Journal of Applied Economics, 1(1), 35-54.

Cameron, A. C., Gelbach, J. B., \& Miller, D. L. (2011). Robust inference with multiway clustering. Journal of Business E' Economic Statistics, 29(2), 238-249.

Coibion, O., \& Gorodnichenko, Y. (2015). Information rigidity and the expectations formation process: A simple framework and new facts. American Economic Review, 105(8), 2644-2678. 
Corsi, F. (2009). A simple approximate long-memory model of realized volatility. Journal of Financial Economic, 7(2), 174-196.

De Resende, C. (2014). An assessment of IMF medium-term forecasts of GDP growth. IEO Background Paper No. BP/14/01, Washington: Independent Evaluation Office of the IMF.

Dovern, J., Fritsche, U., Loungani, P., \& Tamirisa, N. (2015). Information rigidities: Comparing average and individual forecasts for a large international panel. International Journal of Forecasting, 31(1), $144-154$.

Dovern, J., \& Weisser, J. (2011). Accuracy, unbiasedness and efficiency of professional macroeconomic forecasts: An empirical comparison for the G7. International Journal of Forecasting, 27(2), 452-465.

Driscoll, J. C., \& Kraay, A. C. (1998). Consistent covariance matrix estimation with spatially dependent panel data. The Review of Economics and Statistics, 80(4), 549-560.

Easterly, W. (2012). The role of growth slowdowns and forecast errors in public debt crises. In Fiscal policy after the financial crisis (pp. 151-173). University of Chicago Press.

Edwards, S. (2004). Thirty years of current account imbalances, current account reversals, and sudden stops. IMF Staff Papers, 51(s1), 1-49.

Frankel, J. (2011). Over-optimism in forecasts by official budget agencies and its implications. Oxford Review of Economic Policy, 27(4), 536-562.

Greenwood, R., \& Shleifer, A. (2014). Expectations of returns and expected returns. Review of Financial Studies, 27(3), 714-746.

Heston, A., Summers, R., Nuxoll, D., \& Aten, B. (1995). Penn World Tables 5.6. Center for International Comparisons at the University of Pennsylvania (CICUP).

Heymann, D., \& Sanguinetti, P. (1998). Business cycles from misperceived trends. Economic Notes - Siena, 27, 205-232.

Ho, G., \& Mauro, P. (2016). Growth - now and forever? IMF Economic Review, 64(3), 526-547.

Loungani, P. (2001). How accurate are private sector forecasts? Crosscountry evidence from consensus forecasts of output growth. International Journal of Forecasting, 17(3), 419-432.
Marcellino, M., Stock, J. H., \& Watson, M. W. (2006). A comparison of direct and iterated multistep AR methods for forecasting macroeconomic time series. Journal of Econometrics, 135(1-2), 499-526.

Milesi-Ferreti, G. M., \& Razin, A. (1996). Current account sustainability: selected East Asian and Latin American experiences. Working paper No. w5791, National Bureau of Economic Research.

Mincer, J. A., \& Zarnowitz, V. (1969). The evaluation of economic forecasts. In Economic forecasts and expectations: Analysis of forecasting behavior and performance (pp. 3-46). NBER.

Obstfeld, M., \& Rogoff, K. (2007). The unsustainable US current account position revisited. In G7 Current Account Imbalances: Sustainability and Adjustment (pp. 339-376). University of Chicago Press.

Pesaran, M. H., Schuermann, T., \& Smith, L. V. (2009). Forecasting economic and financial variables with global VARs. International Journal of Forecasting, 25(4), 642-675.

Pons, J. (2000). The accuracy of IMF and OECD forecasts for G7 countries. Journal of Forecasting, 19(1), 53-63.

Pritchett, L., \& Summers, L. H. (2014). Asiaphoria meets regression to the mean. Working paper No. w20573, National Bureau of Economic Research.

Reinhart, C. M., \& Reinhart, V. R. (2008). Capital flow bonanzas: an encompassing view of the past and present. Working paper No. w14321, National Bureau of Economic Research.

Sachs, J. (1981). The current account and macroeconomic adjustment in the 1970s. Brookings Papers on Economic Activity, 1(1), 201-268.

Sims, C. A. (2003). Implications of rational inattention. Journal of Monetary Economics, 50(3), 665-690.

Timmermann, A. (2007). An evaluation of the World Economic Outlook forecasts. IMF Staff Papers, 54(1), 1-33.

Woodford, M. (2009). Information-constrained state-dependent pricing. Journal of Monetary Economics, 56, S100-S124. 